\title{
Carbon Nanomaterials in the Treatment of Infectious Bone Defects and Wound Scars after Wushu Fractures
}

\author{
Shenghui Wei, ${ }^{1}$ Lei Zou $\mathbb{D}^{2},{ }^{2} \mathrm{Xu} \mathrm{Hu}{ }^{3}$ and Qiuming $\mathrm{Li}^{4}$ \\ ${ }^{1}$ College Chongqing Normal University School of Physical Education and Health Sciences, Chongqing 401331, China \\ ${ }^{2}$ Wuhan Engineering Institute, Wuhan 430080, Hubei, China \\ ${ }^{3}$ Department of Military Basic Training and Force Management of Army Medical University, Chongqing 400038, China \\ ${ }^{4}$ Panlong Primary School, Jiulongpo District, Chongqing 400051, China
}

Correspondence should be addressed to Lei Zou; zoulei456@163.com

Received 10 August 2020; Revised 18 September 2020; Accepted 25 September 2020; Published 22 October 2020

Academic Editor: Tifeng Jiao

Copyright (c) 2020 Shenghui Wei et al. This is an open access article distributed under the Creative Commons Attribution License, which permits unrestricted use, distribution, and reproduction in any medium, provided the original work is properly cited.

\begin{abstract}
Although modern antibiotics and surgical technology have made great progress, when using carbon nanomaterials to treat bone marrow-induced inflammation after martial arts fractures, how to simultaneously repair bone defects and control wound infections is the current focus of orthopedics research. This paper uses electrospinning technology to develop a carbon nanomaterial based on PLA, HA-g-PLA, and vancomycin. The surface morphology, biocompatibility, drug release, and osteogenesis of carbon nanomaterials are studied, selecting animal models to verify its effect in the treatment of osteomyelitis with bone defects and provide new ideas and new methods for the treatment of bone defects complicated by osteomyelitis infection. In this paper, carbon nanofibers containing doxycycline, a small molecule protease inhibitor, were prepared by simple blending. Encapsulation of carbon nanofibers can control the slow release of doxycycline and improve the effect of doxycycline in treating chronic wounds. This article uses two methods to prepare different types of osteomyelitis models and compare them. After injecting saline or bacterial solution, the two groups were sealed with bone wax and the incision was closed; the blank group did not do any treatment. Within 30 days after surgery, the appearance of the left hind limb wound and general signs of infection were closely monitored, body temperature was measured, and blood was collected from the ear veins of experimental animals to analyze the changes in C-reactive protein (CRP) and procalcitonin levels (PCT); X-ray, CT imaging, and histological observation were performed on 14 and 28 days. Studies have shown that when the drug loading of doxycycline increases from $10 \%$ to $15 \%$, this is related to the change in properties of the polylactic acid fiber membrane from hydrophobic to hydrophilic caused by the increase in doxycycline drug loading.
\end{abstract}

\section{Introduction}

In recent years, with the development of social transportation, industry and construction, traffic accidents, and industrial accidents have increased, and the severity and complexity of physical injuries have become higher and higher. It is reported that after martial arts training, osteomyelitis is more likely to develop. This type of injury has large wounds and heavy pollution, coupled with improper early first aid techniques, improper application of internal fixation techniques, etc., which can easily lead to delayed fracture healing and bone and soft tissue infections and gradually develop into chronic osteomyelitis, skin defects, and sinus formation. Osteomyelitis persists, bone necrosis leaks out, or after repeated surgical debridement, necrotic bone removal, and other reasons, martial arts personnel have bone defects and short limbs. Infective bone defects cannot be cured for a long time, and patients have to bear multiple pressures such as disease pain and economic and mental pressure. This is one of the most complex, tricky, and challenging problems in the orthopedics field.

In the study of foreign researchers in the treatment of infectious bone deficiency after fracture, Soichi randomly divided the rabbits into groups A, B, and C. Group A used TBC and BC to repair bone defects, Group B used TBC combined with $\mathrm{BC}$ and bBMP to repair, and Group $\mathrm{C}$ used 
TBC combined with $\mathrm{BC}$, $\mathrm{bBMP}$, and $\mathrm{AB}$ to repair. The conclusion shows that $\mathrm{TBC}$ is a good carrier for $\mathrm{AB}$ and bBMP. The carbon nanocomposite material can slowly release $\mathrm{AB}$ and $\mathrm{bBMP}$ at its implantation site [1]. Park retrospectively analyzed the bacteriological characteristics of 32 patients with postoperative chronic infection of femoral shaft fractures and the difficulty and treatment strategies of postoperative femoral shaft fractures. All patients underwent debridement, drainage, internal fixation, and bone extension. Results of 32 cases of infection were confirmed by bacteriological examination before operation, including 20 cases of Staphylococcus aureus [2]. Papadia used induction membrane technology to treat 36 patients with infectious bone defects in 37 cases of bone disease, 28 males and 8 females, with an average age of 36 years (range 20-68 years). In the first stage, after removing the internal fixator (for patients with internal fixation), debridement of the infected and necrotic bone tissue and surrounding soft tissues was completed, and the bone defect was repaired [3].

In related domestic research, Guo has developed a new type of antibacterial bone graft substitute that can repair bone defects and inhibit related infections at the same time. This bone composite is prepared by introducing vancomycin (VCM) into nano-hydroxyapatite/collagen/calcium sulfate hemihydrate (nHAC/CSH). The results showed that the $\mathrm{VCM} / \mathrm{nHAC} / \mathrm{CSH}$ composite material performed well in terms of antibacterial ability and bone regeneration. This new type of bone graft substitute should be very promising in the treatment of bone defect-related infections in bone surgery [4]. In order to study how carbon nanomaterials affect infected bone defects, a $3 \mathrm{~mm}$ diameter skull defect infected by methicillin-resistant Staphylococcus aureus (MRSA) was created in adult female Sprague Dawley rats. Micro-CT analysis showed that the newly formed bone volume fraction (BV/TV) in the defect treated with gelatin/ silver significantly increased [5].

In this paper, carbon nanofibers containing both protein and protease inhibitor were prepared. The synergistic effect of protein and protease inhibitor can improve the therapeutic effect of carbon nanofibers on chronic wounds. The preloaded protein chitosan nanoparticles were dispersed into a solution of doxycycline and polylactic acid in hexafluoroisopropanol, electrospinning was performed, and the preloaded particles and doxycycline in the carbon nanofibers were simultaneously embedded inside the fiber. The preloaded particles and doxycycline were uniformly distributed inside the fiber; the protein was continuously released for more than four weeks without burst release, and doxycycline was continuously released for more than two weeks; nanofibers have good cytocompatibility and support cell adhesion and spreading; when carbon nanofibers prepared by this method that simultaneously delivered epidermal growth factor and doxycycline were used for the treatment of back wounds in diabetic rats, they can obtain better treatment than nanofibers that only contain a single drug effect.

\section{Carbon Nanomaterials in the Treatment of Infectious Bone Defects and Wound Scars after Wushu Fractures}

\subsection{VAN/PLGA Microsphere $\beta$-TCP Composite Carbon Nanoscaffold for the Treatment of Infectious Bone Defect after Martial Arts Fracture}

2.1.1. Construction of VAN/PLGA Microsphere $\beta-T C P$ Composite Carbon Nanoscaffold. $10 \mathrm{ml}$ of chitosan solution was prepared with a concentration of $2 \%$ with $1 \%$ glacial acetic acid solution. A dropper was used to soak the material slowly and evenly. It was placed in a $37^{\circ} \mathrm{C}$ oven to dry. This operation was repeated 3 times to make the triphosphate blank stent coated with a layer of positively charged chitosan; the chitosan-coated scaffold was put into a centrifuge tube, and the negatively charged VAN/PLGA sustainedrelease microspheres were dropped in distilled water suspension at the same time and gently shaken, and slightly shake and mix for 10 minutes. The balls were uniformly adsorbed and filled in the pores of the scaffold and centrifuged at $10 \mathrm{rpm}$ for 5 minutes, and the material was taken out and freeze-dried; then the scaffold loaded with microspheres was soaked in the chitosan solution, and after it is completely soaked and saturated, it was taken out and freeze-dried $[6,7]$.

2.1.2. Determination of Porosity of Blank Scaffold, TCS, and EACS. The porosity of the scaffold refers to the percentage of the pore volume of the scaffold in the apparent total volume of the scaffold. The blank sample can be calculated according to the actual parameters of $3 \mathrm{D}$ printing. The apparent volume of the blank sample is $V 0=6 \mathrm{~m} \times 6 \mathrm{~m} \times 5 \mathrm{~m}$, and the material is absolutely dense. The volume of the blank scaffold is $V 1$, and the volume of composite carbon nanoscaffold is $V 2[1,8]$. The absolute compact volume of the stent was measured by the pycnometer method. Under the environment of $30^{\circ} \mathrm{C}$, the pycnometer was filled with absolute ethanol and the sample was weighed as $W 1$. The composite carbon nanoscaffold sample with mass $W 0$ was immersed in the pycnometer filled with absolute ethanol, and vacuum was applied to make the bubbles disappear completely and make anhydrous ethanol fill the pores of the sample, and the sample was weighed as W2 after being filled with absolute ethanol. The sample was taken out and weighed again as $W 3$, and the experimental data were recorded. The blank scaffold and composite carbon nanoscaffold samples were repeated three times each, and the porosity of the composite sample $(n)$ was calculated according to the following formula $(n=\rho /(1+\rho)) . \rho$ is the density of absolute ethanol at $30^{\circ} \mathrm{C}\left(\rho=0.78 \mathrm{~g} / \mathrm{cm}^{3}\right)$.

2.1.3. Determination of Sustained Release of Drugs in TCS and EACS. 3 TCS and EACS of equal quality were randomly 
taken and put in a $10 \mathrm{ml}$ test tube, $1 \mathrm{ml}$ dichloromethane was added to fully dissolve the microspheres in the composite carbon nanoscaffold, and $4 \mathrm{ml}$ PBS solution was added to the test tube and centrifuged to take the supernatant. The absorbance at $260 \mathrm{~nm}$ was measured in a spectrometer, and the average value was repeated 3 times, and the mass of VAN contained in the composite carbon nanoscaffold was calculated using the standard curve $[9,10]$. Then, 3 TCS and EACS of equal quality were randomly taken, respectively, and $50 \mathrm{ml}$ PBS (pH 7.4) solution was added, sealed, and placed in a constant temperature plate shaker at $37^{\circ} \mathrm{C}$, and shaken at a constant speed, and $0.5 \mathrm{ml}$ was taken out at $1 \mathrm{~d}$, $2 \mathrm{~d}, 3 \mathrm{~d}, 6 \mathrm{~d}, 12 \mathrm{~d}$, and $24 \mathrm{~d}$. The solution was stored for testing and replaced with $0.5 \mathrm{ml}$ PBS. After the sample was centrifuged, the supernatant was taken, and the absorbance at $260 \mathrm{~nm}$ was detected by an ultraviolet spectrophotometer. The drug concentration was calculated using the standard curve, and the average value was used to calculate the cumulative drug release and sustained release rate.

\subsection{Carbon Nanomaterials Treat Wounds Caused by Martial} Arts Fractures. Nanofibers will be ejected from a place on the Taylor cone where the electrostatic force of the liquid is greater than the surface tension. When the fiber passes through the air after being ejected, the solvent evaporates quickly and deposits on the receiver $[11,12]$. Although the research of electrospinning technology has been going on for a hundred years, the use of this cheap manufacturing process of nano- or microfiber is still attracting attention. At present, many natural and synthetic polymers can be made into nanofibers by electrospinning.

2.2.1. Tissue Engineering. Electrospun polyester carbon nanofibers have the characteristics of extremely high porosity, large specific surface area, and interconnected pores. These characteristics make it mimic the extracellular matrix to a certain extent and provide the necessary environment for cell proliferation and differentiation. In addition, the morphology and mechanical properties of the fiber can be controlled by adjusting the system parameters (such as the molecular weight of polyester and solution properties) and process parameters (such as flow rate, voltage, and distance between needle and receiver) in the electrospinning process. The morphology and mechanical properties of the fiber can be controlled by adjusting the system parameters (such as the molecular weight of polyester and solution properties) and process parameters (such as flow rate, voltage, and distance between the needle and the receiver) in the electrospinning process to meet biological requirements, i.e., engineering needs $[13,14]$. Currently, electrospun polyester carbon nanofibers have been reported to be used to construct tissue engineering scaffolds such as cartilage, skin, bone, nerves, and blood vessels.

2.2.2. Drug Delivery. Electrospun polyester carbon nanofibers for drug delivery have the characteristics of high drug loading, good biocompatibility, and degradability. At the same time, the size and degradation rate of nanofibers can be controlled by changing the parameters in the electrospinning process, thereby adjusting the drug release rate in the body.

2.2.3. Wound Healing. Electrospun polyester carbon nanofibers also have unique advantages as trauma dressings. The pore size of the electrospun polyester carbon nanofiber membrane is usually in the nanometer level, while the size of the bacteria is in the micron level, which can resist the invasion of external bacteria into the wound, thereby preventing wound infection and promoting wound healing $[15,16]$. At the same time, because the electrospun polyester carbon nanofiber membrane has a higher porosity and a larger specific surface area, as a wound dressing, on the one hand, it can ensure the penetration of water and gas on the wound surface, so as to keep the wound surface moist. The proliferation and differentiation provide nutrients; on the other hand, it can quickly absorb the tissue fluid secreted by the wound, which is conducive to the coagulation of blood, keeps the surface of the wound clean, and promotes the repair of the wound. From the perspective of the patient, electrospun polyester carbon nanofibers as a trauma dressing can reduce the formation of scar tissue and the frequency of dressing changes, thereby reducing the mental and physical pressure of the patient $[17,18]$.

In view of the advantages of polyester nanofibers in drug delivery and wound healing, many drugs are encapsulated in the fibers to construct bioactive wound dressings for the treatment of chronic wounds. These drugs include growth factors, antibiotics, and vitamins.

\subsection{Surgical Methods}

2.3.1. Debridement and Soft Tissue Treatment. The patient was placed in a supine position, disinfected, and draped after general anesthesia intubation. During the operation, the sinus, infected tissue, and soft tissue defect surrounding necrotic fascia and inflammatory tissue were removed from the wound to ooze blood. Infected bone, dead bone, and hardened bone were removed. For those who have undergone internal fixation, the internal fixation material needs to be removed completely, and the inflammatory granulation tissue under the steel plate and in the medullary cavity should be scraped off to open the closed medullary cavity $[19,20]$. The two fractured ends of the bone defect were repaired with an electric saw, resulting in "spot hemorrhage" on the surface of the cortical bone, and the bone was pale red. Try to protect the periosteum around the fracture. Part of the scar tissue and bone fragments were sent for examination and bacterial culture during the operation. The surgical wound was continuously flushed with hydrogen peroxide solution and $0.9 \%$ sodium chloride solution, soaked in the diluted iodophor solution for 10 minutes, and flushed again with $0.9 \%$ sodium chloride solution. Under normal circumstances, after debridement, the wound can be sutured to cover the broken end of the bone. The wound does not need to be sutured forcibly. Open dressing can be 
given to avoid skin necrosis due to excessive skin tension $[21,22]$. If the wound is combined with exposed tendons, combined with skin flap transfer to repair the wound, the choice of the flap can be based on the situation. Adjacent fasciocutaneous flap, cutaneous nerve nutrient vessel flap, or free skin flap can be used.

2.3.2. Osteotomy. Before the osteotomy, the iodophor gauze covering the wound bread should be tied up to protect the surgical field, the gloves were changed, and the osteotomy area was disinfected again to prevent contamination of the osteotomy site. The location of the osteotomy is determined according to the location of the bone defect and the length of the bone segment. Generally, the distal femur and the proximal metaphysis were selected, away from the lesion, and the location with good blood supply and healthy skin was selected. A small incision was used at the metaphyseal osteotomy during the operation to protect the periosteum. The $2.5 \mathrm{~m}$ Kirschner wire was used for low-energy osteotomy under a special minimally invasive osteotomy sleeve [23, 24]. During the operation, a C-arm X-ray machine can be used to assist the fluoroscopy positioning of the osteotomy site. After the osteotomy was completed, the severed end was appropriately pressurized to help stop bleeding and promote the rapid formation of callus. The timing of osteotomy was determined by the degree of wound infection. If the infection is mild, an external fixation frame, metaphyseal osteotomy, open wound dressing, and iodophor gauze fill the wound cavity. This method was used in 12 patients in this group. If the infection is serious, debridement of the wound was completed in the first stage, an external fixator is fixed, and osteotomy was performed 3 to 6 weeks after infection control. The 5 cases in this group were severely infected and were treated in two phases.

2.3.3. Postoperative Treatment. The sensation, movement of the affected limb, and blood supply at the end of the toe after the operation were closely observed. The affected limb was elevated to reduce swelling. According to the results of bacterial culture, sensitive antibiotics were routinely applied for 4 weeks, the dressing was changed according to the wound condition 2 to 3 days after the operation, pay attention to aseptic operation, the dressing was changed every day or every other day, and the wound was filled with iodophor gauze. Patients were encouraged to do leg-lift training and exercise for muscle isometric contraction. The active and passive activities of adjacent joints were strengthened, the muscles of the lower limbs were trained, and further contractures of the hip and knee joints were avoided. After 5 days after operation, they can walk with crutches and part of the weight-bearing to give stress stimulation to the new bone. Antibiotics are routinely used after surgery. In order to keep the needle track clean, the needle track needs to be cleaned with saline and disinfected with alcohol every day. Strengthening needle tract care can effectively prevent needle tract infections.

The osteotomy began to be extended 7 to 10 days, and the stretch was extended at $1 \mathrm{~m} / \mathrm{d}, 0.25 \mathrm{~mm}$ each time, in 2 to 4 times. During the bone removal process, the frontal and lateral X-rays of the affected limb shall be taken every 2 weeks to observe the prolongation and extension of the osteotomy, whether there is axial offset, etc., and the changes in the length of the affected limb were observed, and the bone lengthening speed and lower limb force line in time were adjusted. If the bone growth at the osteotomy site is poor, the transportation should be stopped in time or retracted by $2 \mathrm{~cm}$, and the callus should be observed for 1 to 2 weeks to continue the transportation. In the process of stretch extension, if skin stretch pain occurs, oral analgesics can be used to relieve it. If the relief is not obvious or if the pain symptoms worsen, the speed of stretch should be appropriately slowed down. During the follow-up, the healing of the femur of the affected limb, the sensation, movement, and function of the affected limb were observed. Follow up on time after the operation and reasonably guide them to perform functional exercises, get out of bed as soon as possible under the guidance of the rehabilitation doctor, and gradually move from partial weight-bearing activities to full-weight exercises. When the affected limb is stretched to align with the fractured end of the bone defect and is the same length as the contralateral limb, the extension or bone movement is stopped. Axial compression is applied once every 2 weeks, and the contact surface stress is stimulated by $1 \mathrm{~mm}$ each time to promote bone fracture.

According to the reexamination of X-rays, the new bone mineralization is complete and the bone defect ends completely reach the clinical healing standard, and the external fixation needle and connecting rod can be removed in stages. Strenuous exercise should be avoided within half a year after the external fixator is removed to avoid refracture of the affected limb. If there are lines of force, abnormal alignment, and nonunion of the fractured ends of the bone defect, it is necessary to adjust the line of force and alignment of the femur, and the fractured end was fixed with bone grafting and compression to promote its healing. Great attention should be paid to postoperative follow-up, to strengthen postoperative management and communication with the affected limb and to establish patient confidence in treatment.

\section{Experimental Study of Carbon Nanomaterials for Treating Infectious Bone Defects and Wound Scars after Martial Arts Fractures}

\subsection{Preoperative Preparation and Postoperative Treatment}

3.1.1. Preoperative Preparation. After admission, the patient's sinus wounds dressings should be changed immediately. When dressings were changed, secretions were taken from the depths of the wounds, and bacterial culture and drug sensitivity experiments were performed. While selecting sensitive antibiotics, the overall condition of patients with infectious nonunion is evaluated. Most patients with infectious nonunion have been in bed for a long time and have undergone multiple operations. Some patients have repeated infections and have poor general conditions. It 
can improve the heart and lung function, enhance the patient's endurance for surgery, and improve related muscle strength, joint function, and osteoporosis. Determine whether the patient has systemic diseases such as diabetes and anemia. At the same time, it is necessary to evaluate the local soft tissue condition of the injured limb, the location and type of fracture, the size of the bone defect, the wound condition around the bone defect, and the condition of the original fixation.

3.1.2. Postoperative Treatment. Strict aseptic operation should be maintained during dressing change after surgery to prevent reinfection of the wound. Antibiotics were applied once before surgery and for 2 days after surgery. Depending on the exudation of the wound, the wound is usually opened 2 days after the operation, the dressing is changed, and then the dressing is changed every other day. When the dressing is changed, part of the exudate and blood clot on the wound surface is removed. If the granulation tissue formation is slow, scraping can be used. Scrape the spoon gently until the wound oozes blood, apply gentamicin mixed with saline keep the moist environment covering the wound, and the outer layer shall be bandaged with sterile gauze. After the wound granulation tissue is fresh, the wound can be sealed by flap transfer or free skin grafting.

\subsection{Double Drug Loading of Electrospun Carbon Nanofibers.} The method of encapsulating protein and protease inhibitor mentioned in this article simultaneously encapsulates two drugs. First, a hexafluoroisopropanol solution of polylactic acid was prepared (concentration of $2 \mathrm{wt} . \%$ ). Then, a certain amount of preloaded protein chitosan nanoparticles and doxycycline were mixed with the above polylactic acid solution so that the mass content of the preloaded particles was 9.1 wt.\% and the drug loading amount of doxycycline between 10 and $15 \%$, the mixture was stirred for 1 to 3 hours to make the preloaded particles and doxycycline uniformly dispersed in the solution. Next, the mixture was transferred into a $1 \mathrm{~mL}$ glass syringe connected to a 12-gauge stainless steel needle and spun at $15 \mathrm{kV}$. An aluminum plate with a diameter of $10 \mathrm{~cm}$ was used to receive nanofibers at a distance of $15 \mathrm{~cm}$ from the needle.

3.3. Porosity, Mechanical Properties, Drug Sustained Release Determination, and Data Collection of TCS and EACS. The blank sample volume is $=6 \mathrm{~m} \times 6 \mathrm{~m} \times 5 \mathrm{~m}$, trabecular $=40 \mu \mathrm{m}$, aperture $=50 \mu \mathrm{m}$. After measurement, the actual porosity of the blank stent is $48 \pm 1.2 \%$; the porosity of TCS is $25.46 \pm 2.45 \%$; the porosity of EACS is $21.9 \pm 0.79 \%$.

The maximum bearing capacity of TCS material measured by universal material mechanics is 215.9 $\mathrm{N} \pm 12.4 \mathrm{~N}$; the maximum bearing capacity of EACS material is $21.5 \mathrm{~N} \pm 9.7 \mathrm{~N}$. According to the formula: $1 \mathrm{~Pa}=1 \mathrm{~N} /$ square meter, the support area of the stent material is $36 \mathrm{~m}^{2}$ and the conversion strength is about
$6 \mathrm{MPa}$ (much higher than the minimum compressive strength of cancellous bone $2 \mathrm{MPa}$ ).

In vitro drug release experiments were performed on TCS and EACS. In order to simulate the release of composite stents in vivo to the maximum, we conducted drug release experiments in $\mathrm{PBS}$ solution at $37^{\circ} \mathrm{C}$. The figure shows the VAN/PLGA microspheres, TCS, and EACS. The figure shows the release of VAN in VAN/PLGA microspheres, TCS, and EACS. The first $3 \mathrm{~d}$ VAN microspheres and TCS both had burst releases to varying degrees. However, in EACS, the burst release of chitosan coating was significantly reduced. After $3 \mathrm{~d}$, the drug release of each group was slow and stable, and the drug release rate at $24 \mathrm{~d}$ was about $70 \%$.

\subsection{Immunohistochemical Detection of BMP-2 and VEGF} Expression. Using the SP (streptavidin-peroxidase) method, that is, the streptavidin-peroxidase linkage method, the nucleus and/or the cytoplasm of light brown particles, brown particles, and yellow brown particles, the expression is a positive expression, and no particles are attached, or only a chip or yellow staining is regarded as a negative expression. The expression is a positive expression, and no particles are attached, or only a chip or yellow stain is regarded as a negative expression. In this experiment, an Olympus optical microscope was used to take pictures and for observation. First, the bone tissue expression site was located through a $40 \mathrm{x}$ low-power lens, and then the absorbance value of the specific expression site was determined at 400x. The microscopic imaging system uses 8 Olympus CCDP73 to collect immunohistochemical pictures of bone tissue. 10 400x fields of view were randomly selected for each slice and Image Pro 6.0 software was used to calculate the average absorbance value and read the positive expression rate.

\section{Experimental Research and Analysis of Carbon Nanomaterials in the Treatment of Infected Bone Defects and Wound Scars after Martial Arts Fractures}

4.1. TCS and EACS Load BMSCs Culture. BMSCs were seeded on blank scaffolds, TCS, and EACS and cultured for 7 days. Observed by scanning electron microscope, it was found that the three scaffolds all had cell proliferation, and the outer surface was covered with long flat cells. The CCK8 experimental analysis showed that BMSCs on these three scaffolds increased with the extension of the culture time, and these three scaffolds were all conducive to cell replication. The experiment is shown in Figure 1.

As shown in Figure 1, the number of cells in the blank scaffold was less on the $1 \mathrm{~d}$, and the difference between the blank scaffold and the composite scaffold was statistically significant $(P<0.05)$, and the number of cells on the blank scaffold was slightly more than that on the 3rd, 5th, and 7th days. Compared with TCS and EACS, blank stents on the 3rd and 5th days of TCS and EACS had statistically significant differences $(P<0.05)$. The growth trend of TCS and EACS groups was roughly the same, and the comparison was not statistically significant $(P>0.05)$. 


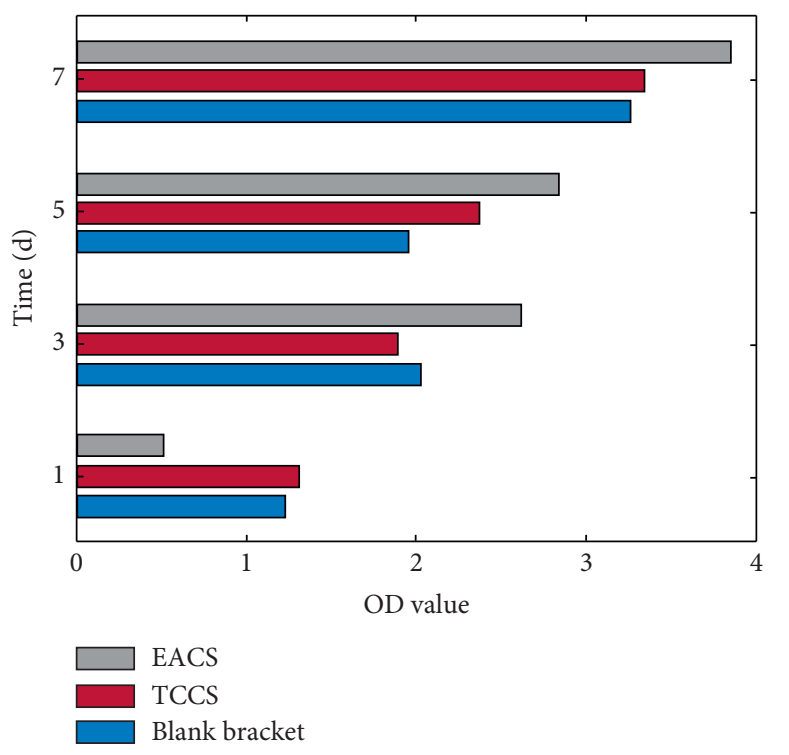

FIGURE 1: Proliferation of BMSCs on blank stents, TCS, and EACS.

4.2. Analysis of Postoperative Improvement. During the follow-up period, there was no refracture of new bone and no secondary hip dislocation. 7 cases of needle tract infections were given oral antibiotics, active dressing changes, and needle eye debridement and suture, and the infection was cured. Three cases showed axial deviation during bone lengthening, which was gradually corrected after adjustment by external fixator. 9 patients with skin traction pain were given oral analgesics to slow down the traction speed and improve the effect, as shown in Table 1.

According to the Paley fracture healing scoring standard, the excellent and good rate was $82.4 \%$. At the last follow-up, the scores of all dimensions of the SF-36 scale were significantly higher than those before the operation $(P<0.05)$, the difference was statistically significant, and the patient's physical and mental health levels were comprehensively improved. Through functional exercises after surgery, the knee joint extension and flexion function were improved compared to before surgery, and normal walking was not affected. All patients were very satisfied with the treatment results.

4.3. VEGF Test Results. By comparing the VEGF expression levels of the four groups at 1 week, 2 weeks, and 4 weeks after surgery, at each time point between group A and group B, and group $C$ and group $\mathrm{D}$, there was no significant difference in VEGF expression $(P>0.5)$. Histogram of VEGF expression at 1,2 , and 4 weeks after surgery is shown in Figure 2.

The difference between the expression levels of the groups A and B and the expression levels of the groups $C$ and $\mathrm{D}$ is statistically significant. The expression level of VEGF in each group at the three time points was statistically significant $(P<0.5)$, and the expression level of VEGF in the four groups was the highest in the second week.
TABle 1: Comparison of SF-36 scale scores of 19 patients with femoral infectious bone defect before operation and last follow-up.

\begin{tabular}{lcccc}
\hline Project & $\begin{array}{c}\text { SF-36 scale score }(X \pm S, \\
\text { points })\end{array}$ & $\begin{array}{c}t \\
\text { value }\end{array}$ & $\begin{array}{c}P \\
\text { value }\end{array}$ \\
& Preoperative & Postoperative & & \\
\hline $\begin{array}{l}\text { Physiological } \\
\text { function }\end{array}$ & $45.37 \pm 3.56$ & $68.36 \pm 4.29$ & 13.28 & $<0.05$ \\
Body pain & $36.39 \pm 12.37$ & $58.13 \pm 13.56$ & 12.39 & $<0.05$ \\
Mental health & $42.38 \pm 5.38$ & $58.38 \pm 5.32$ & 15.34 & $<0.05$ \\
\hline
\end{tabular}

4.4. Results of Fracture Healing and Functional Recovery. Patients in the microparticle group were followed up regularly for 3 to 48 months after surgery, with an average follow-up of 24 months. VSD was placed in 25 patients for granulation culture after surgery. The average survival time of granulation was 9.5 days. The bone graft is covered by granulation tissue for an average of 7 weeks after the microgranular bone graft. Flap transfer or simple skin grafting surgery was used to fill the wound. The fractures of all patients healed with an average healing time of 6 months (3-8 months). After an average of 7 months (4-12 months) after surgery, no bone abnormalities were found, and the external fixator was removed. Bone healing and functional recovery are good. Comparison of bone healing between the two groups is shown in Figure 3.

Patients in the traditional granule group were followed up regularly for 9 months to 26 months after surgery, with an average follow-up of 19 months. Twenty-seven patients were also implanted with ordinary granular bone after the granulation survived. The bone has been covered by granulation tissue for an average of 9 weeks after bone grafting. Fill the wound with further bone grafting. One patient reinfected after operation and healed completely after retreatment. The two groups of patients recovered well after the operation and were discharged. According to the Ilizarov method, the fracture healing and functional recovery of the two groups reached satisfactory results. There was no significant difference and no statistical significance $(P>0.05)$.

4.5. Release Behavior of Two Drugs. Figure 4 shows the release behavior of doxycycline from dual drug nanofibers with a drug loading of $10 \%$ and $15 \%$. Nanofibers containing only doxycycline served as a control. It can be seen that when the drug loading amount of doxycycline is $10 \%$, after two weeks of culture, the carbon nanofibers containing only doxycycline released $24 \%$ of the total doxycycline, while the dual drug nanofibers released $5 \%$ of doxycycline. The experiment is shown in Figure 4 when the drug loading of doxycycline is $15 \%$.

After two weeks of culture, the carbon nanofibers containing only doxycycline released $34 \%$ of the total doxycycline, while the dual drug nanofibers released $62 \%$ of the doxycycline, and the latter had obvious burst. Simultaneous protein encapsulation speeds up the release of doxycycline, which may be because the chitosan particles used for protein preencapsulation are more hydrophilic than 


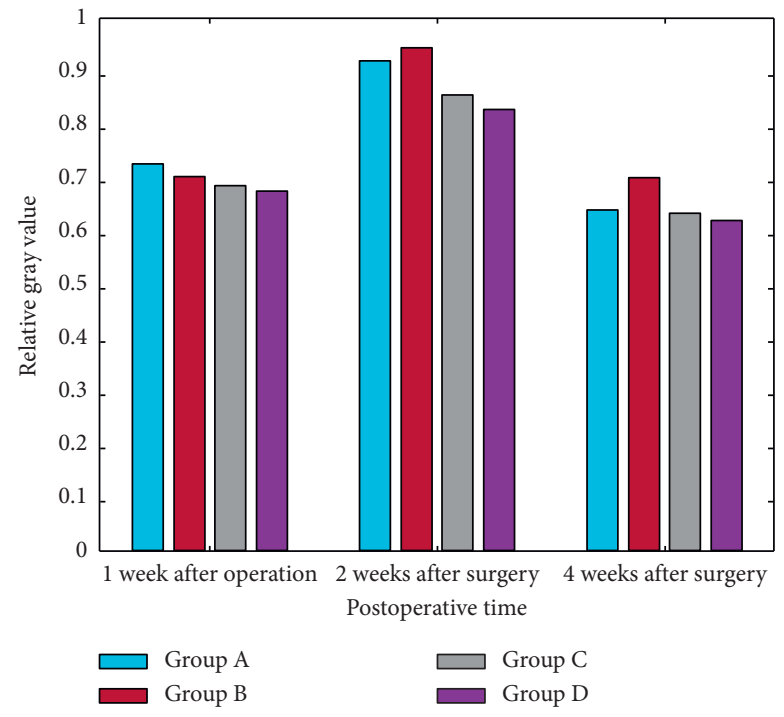

Figure 2: Histogram of VEGF expression at 1, 2, and 4 weeks after surgery.

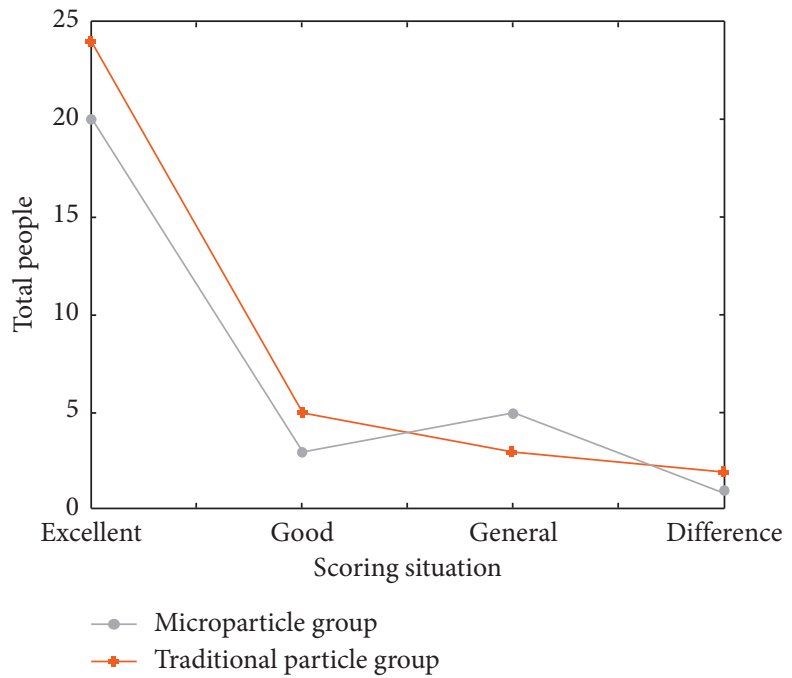

Figure 3: Comparison of bone healing between the two groups.

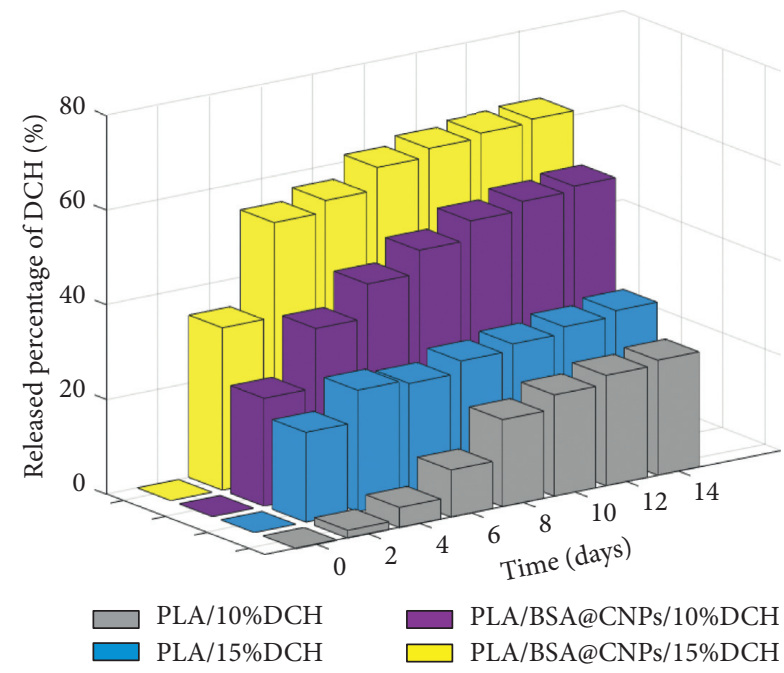

Figure 4: Release behavior of doxycycline. polylactic acid, which is more conducive to the diffusion of doxycycline chitosan. When the drug loading of doxycycline increases from $10 \%$ to $15 \%$, its release rate from the dual drug nanofibers increases. This should be related to the increase in doxycycline drug loading that causes the nanofiber membrane to change from hydrophobic. It is related to changes in hydrophilic properties. Water molecules enter the hydrophilic fibers more easily, allowing the doxycycline inside to diffuse out faster.

\section{Conclusions}

Autologous microgranular bone has the characteristics of strong osteogenesis and release of more cytokines. It has been used in clinical treatment of infectious bone defects and nonunion to achieve good results. Autogenous microgranular bone grafting has greater advantages than traditional granular bone grafting in the treatment of infectious 
bone defects and nonunion. Therefore, autologous microparticle bone grafting can improve the efficiency of treating infectious bone defects and is a more active and effective method.

In this paper, a reasonably designed particle preencapsulation method is used to realize the encapsulation and controlled release of protein by carbon nanofibers while maintaining protein activity. The nanofiber has a smooth surface and uniform size. The preloaded particles are evenly distributed in the nanofibers. The protein retains its biological activity and can be continuously released from the nanofibers for more than four weeks without obvious burst release. Nanofibers have good cell compatibility and can support cell adhesion, spreading, and proliferation. When the carbon nanofibers coated with epidermal growth factor prepared by this method are used for the treatment of back wounds of diabetic rats, they obtain better therapeutic effects than blank nanofibers.

Excluding hyperplastic scars and contaminated or poorly healed granulation tissue, eliminating dead space, draining pus and blood, and improving local blood circulation have become one of the important principles for the treatment of infectious bone defects such as chronic osteomyelitis. It is generally believed in clinical practice that any dead space with dead bones, or sinus formation, discharge of pus, or sufficient new bone formation to form a package, should be treated with surgical intervention to achieve clinical healing conditions. Lesion debridement is the basis and effective method for the treatment of infectious bone defects. During debridement, the suspiciously infected tissue should also be completely removed until healthy and bleeding bone is exposed. For the treatment of infectious bone defects, thorough surgical debridement, adequate drainage, and avoiding the formation of dead spaces have become the consensus of many scholars. Among them, the control of infection is still considered to be the most critical link to successfully cure infectious bone defects.

\section{Data Availability}

The data that support the findings of this study are available from the corresponding author upon reasonable request.

\section{Conflicts of Interest}

The authors declare that they have no conflicts of interest.

\section{Acknowledgments}

This study was supported by the 2017 National Social Science Foundation Project "A Study of Bashu Body Graphs" (no. 17BTY013) and Youth Project of Science and Technology Research Program of Chongqing Education Commission of China (no. KJQN202000509).

\section{References}

[1] H. Soichi, M. Akihiko, O. Kotaro et al., "Treatment of mandibular fracture-associated infectious pseudarthrosis accompanied by osteomyeliis in a peritoneal dialysis patient," Journal of UOEH, vol. 40, no. 2, pp. 209-215, 2018.

[2] J. Park and W. Y. Chung, "Osteochondral allograft reconstruction of talar body fracture with a large bone defect," Archives of Orthopaedic and Trauma Surgery, vol. 136, no. 1, pp. 35-40, 2015.

[3] D. Papadia, A. Musetti, and E. Dematte, "An injectable bone substitute eluting antibiotic in the treatment of traumatic bone defects and its prophylactic effect," Georgian Mathematical Journal, vol. 97-B, no. 16, pp. 161-186, 2015.

[4] Z. M. Guo, T. C. Shangguan, M. Zhang et al., "Prevention and treatment of the related complications of tibial fractures bone defect by bone transport," Zhongguo Gu Shang = China Journal of Orthopaedics and Traumatology, vol. 29, no. 8, pp. 756-760, 2016.

[5] L. Zhong, X. Hong, Z. Zhang et al., "Staged treatment of infectious long bone defect in lower extremity," Zhongguo Xiu Fu Chong Jian Wai Ke Za Zhi, vol. 28, no. 10, pp. 1212-1216, 2014.

[6] C. Zongjie and W. Zhan-You, "Additional steel plate and bone graft in the treatment of bone nonunion after intramedullary nail fixation for femoral fractures," Chinese and Forgn Medical Research, vol. 64, no. 6, pp. 433-437, 2015.

[7] Y. Yang, X. Niu, Q. Zhang et al., "Efficacy of bioactive glass and allogenic bone in repair of bone defect after benign bone tumor curettage," Chinese Journal of Reparative \& Reconstructive Surgery, vol. 30, no. 6, pp. 675-679, 2016.

[8] M. J. Rose, P. C. Cevallos, L. Gellis, and L. Bergersen, "Lateterm development of an atrial defect and thrombus formation after device fracture following successful transcatheter closure of an atrial septal defect with a STARFlex device," Cardiology in the Young, vol. 27, no. 5, pp. 975-977, 2017.

[9] W. Bin, J. Song, L. U. Aidong et al., "Bone transportation by ring type external fixator combined with locked intramedullary nail for tibial non-infectious defect," Chinese Journal of Reparative \& Reconstructive Surgery, vol. 29, no. 11, pp. 1348-1352, 2015.

[10] A. M. H. Raphael, A. V. Roberto, R. Alejandro et al., "Management of craniocerebral gunshot injuries: a review," Korean Journal of Neurotrauma, vol. 11, no. 2, pp. 35-43, 2015.

[11] W. Yicun, J. Hui, D. Zhantao et al., "Comparison of monolateral external fixation and internal fixation for skeletal stabilisation in the management of small tibial bone defects following successful treatment of chronic osteomyelitis," BioMed Research International, vol. 2017, Article ID 6250635, 8 pages, 2017.

[12] K. Naito, Y. Sugiyama, H. Obata, A. Mogami, O. Obayashi, and K. Kaneko, "Screw fixation and autogenous bone graft for an irreducible distal ulna fracture associated with distal radius fracture," The Journal of Hand Surgery Asian-Pacific Volume, vol. 22, no. 2, p. 236, 2017.

[13] M. L. Kaneko, C. J. A. Van Bergen, L. Blankevoort et al., "Computed tomography analysis of osteochondral defects of the talus after arthroscopic debridement and microfracture," Knee Surgery, Sports Traumatology, Arthroscopy, vol. 24, no. 4, pp. 1286-1292, 2016.

[14] D. Lin, D. Luo, K. Lian, W. Zhai, and Z. Ding, "Reconstruction of traumatic bone defect with in situ implantation of dropped traumatic segmental bone fragments," Orthopedics, vol. 39, no. 1, pp. e14-e18, 2016.

[15] N. M. Haines, W. D. Lack, R. B. Seymour, and M. J. Bosse, "Defining the lower limit of a "critical bone defect" in open 
diaphyseal tibia fractures," Journal of Orthopaedic Trauma, vol. 30, no. 5, pp. e158-e163, 2016.

[16] H. Wang, W. Jiang, X. Wei, Y. Rui, and Z. Sun, "Ulnius formation for forearm fracture with segmental radial defect," International Journal of Clinical \& Experimental Medicine, vol. 8, no. 10, pp. 17835-17838, 2015.

[17] F. J. M. Verstraete, B. Arzi, D. J. Huey, D. D. Cissell, and K. A. Athanasiou, "Regenerating mandibular bone using rhBMP-2: part 2-treatment of chronic, defect non-union fractures," Veterinary Surgery, vol. 44, no. 4, pp. 410-416, 2015.

[18] J. Silva, C. F. Mourão, H. Rocha Junior, L. F. Magacho da Silva, G. Moraes, and N. Homsi, "Treatment of frontal bone fracture sequelae through inversion of the bone fragment," Revista Do Colegio Brasilro De Cirurgioes, vol. 43, no. 6, pp. 472-475, 2016.

[19] H. Qu, W. Guo, R. Yang et al., "Reconstruction of segmental bone defect of long bones after tumor resection by devitalized tumor-bearing bone," World Journal of Surgical Oncology, vol. 13, no. 1, p. 282, 2015.

[20] Y. Yang, X. C. Ramirez, X. Wang et al., "Impact of carbon nanotube defects on fracture mechanisms in ceramic nanocomposites," Carbon, vol. 115, pp. 402-408, 2017.

[21] Z. Guo, L. Shi, S. Tian, W. Chen, and B. Lin, "Effectiveness of limbs shortening and re-lengthening in treatment of tibial infectious bone defect and chronic osteomyelitis," Zhongguo Xiu Fu Chong Jian Wai Ke Za Zhi = Zhongguo Xiufu Chongjian Waike Zazhi = Chinese Journal of Reparative and Reconstructive Surgery, vol. 31, no. 8, pp. 941-945, 2017.

[22] H. Chen, X. Ji, M. Hao, Q. Zhang, and P. Tang, "A three-stage procedure using bone transportation for the treatment of sternoclavicular infectious arthritis," Journal of Orthopaedic Surgery \& Research, vol. 11, no. 1, p. 152, 2016.

[23] U. S. Gill, A. Zissimopoulos, S. Al-Shamma et al., "Assessment of bone mineral density in tenofovir-treated patients with chronic hepatitis B: can the fracture risk assessment tool identify those at greatest risk?" Journal of Infectious Diseases, vol. 211, no. 3, pp. 374-382, 2015.

[24] L. Burke, C. Yazhou, L. Peijuan et al., "Effect of unilateral producible external fixation apparatus on treating open tibia fracture(Gustilo III B/C) with bone defect," Lingnan Modern Clinics in Surgery, vol. 49, no. 4, pp. 200-212, 2015. 